REVIEW

\title{
GASTRIC STUMP CANCER. ETIOLOGY AND TREATMENT. A GENERAL REVIEW OF THE LAST 5 YEARS
}

\author{
V. Braga ${ }^{1}$, I. Slavu1 ${ }^{1}$ A. Tulin ${ }^{2}$, B. Socea ${ }^{3}$, L. Alecu ${ }^{2}$ \\ ${ }^{1}$ General Surgery Clinic, Emergency Clinical Hospital, Bucharest, Romania. \\ ${ }^{2}$ General Surgery Clinic, "Prof. Dr. Agrippa Ionescu” Emergency Clinical Hospital, Bucharest, Romania. \\ ${ }^{3}$ General Surgery Clinic, Pantelimon Emergency Clinical Hospital, Bucharest, Romania \\ Corresponding author: Slavu Iulian \\ Phone no. 0040212228129 \\ E-mail: iulian.slavu@yahoo.com
}

\begin{abstract}
Gastric stump cancer (GSC) is an entity described in the literature as early as the 1920s. Along the years the clinicopathological characteristic had changed. At the moment, there are no wellestablished guidelines regarding the optimal treatment. We conducted a literature review of the studies published from 2014 to 2019 using the PubMed database. After searching the PubMed database for the keywords: "gastric remnant cancer" AND "stump" AND "neoplasm" we identified 167 articles. Of these, 25 studies were considered relevant. GSC develops after 2 years from the primary intervention for malignancy and after 20 years for benign lesions. The development mechanisms vary depending on the type of primary gastric lesions. GSC is considered a unique clinicopathological entity. The pattern of lymphatic drainage is different in comparison to primary gastric neoplasm. Thus, an important negative prognostic factor is considered to be the $N$ group of TNM staging. More studies are required to improve the understanding of development mechanisms and evolution of this pathology. Survival after gastric cancer has increased and it is possible to observe an increased percentage of patients who achieve the 5-year survival interval after surgery. Currently, there are no guidelines to select the optimal treatment with regards to surgery or chemotherapy as pathology is rare and studies which investigate the evolution and prognosis lack a significant cohort of patients. Therefore, the existent data is not substantial enough to elaborate guidelines that would define a standard surgical treatment.
\end{abstract}

Keywords: gastric stump cancer, treatment, review

\section{Introduction}

The first case of gastric stump cancer (GSC) after a partial gastrectomy was first described by Balfour in 1922. GSC is defined as a neoplasm that develops at the level of the gastric stump, after partial gastric resections conducted for malignant or benign pathology [1]. Although initially this pathology was viewed as a relapse of a primary gastric malignancy, nowadays, it is regarded as a distinct clinicopathological entity with specific treatment and etiology [2].

The prevalence of GSC is low and ranges between $1 \%-3 \%$ of all gastric resections [3]. Frequently, GSC develops after distal gastrectomy performed for malignant tumors [4]. An influential role for this characteristic is attributed to the chronic treatment with proton pump inhibitors and anti-H2 therapy, which have led to a significant decline of surgical procedures 
for benign lesions [5]. Due to a lack of symptoms GSC is diagnosed in an advanced stage when few treatment resources are available and has a low resectability rate and a poor prognosis. The surgical treatment in these is prone to increased morbidity and mortality compared to the index intervention. This results from the modified local anatomy, changes of lymphatic drainage and postoperative adhesions. Also, these patients frequently received chemotherapy and/or radiotherapy for the primary tumor which impacts the nutrition status and overall ability cope with another surgical intervention.

The aim of our study was to evaluate the current knowledge published during the last 5 years with regards to the etiology of GSC, treatment and prognosis. Trends in benign gastric resection have shifted from perforated ulcers which are now mainly treated by proton pump inhibitors to bariatric surgery which frequently implies different types of partial gastric resections which leave in place stumps exposed to develop tumors.

\section{Materials and Methods}

We conducted a literature review to identify the published articles between 2014 and 2019, which studied GSC. We assessed the abstract and full text of all relevant articles. The investigated variables were: number of patients included, clinical and demographic information, TNM staging, types of surgical interventions and outcomes.

The research was performed using the words "gastric remnant cancer" AND "stump" AND "neoplasm". We identified 167 studies, from which 42 were written in the English language and included. After filtering the articles that analyzed the clinicopathologic characteristics of GSC 30 studies were selected. Of these 3 papers were not compatible with the subject and 2 papers had only the abstract published. After this evaluation 25 were used for our analysis. The P.I.C.O.S concept (patient, intervention, comparator, outcome, study type) was used to structure the questions and the research topic as to attain clinical validity. The results of the search were filtered following the PRISMA checklist (Preferred Reporting Items for Reviews and Meta-Analysis) (Figure 1). We used the standard recommendation of two independent readers who performed the selection and subsequent extraction process.

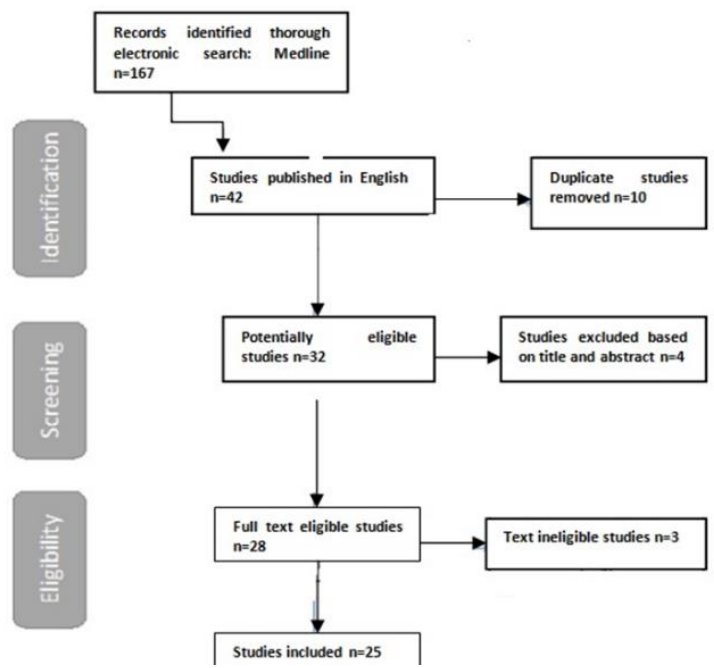

Figure 1 - Analysis protocol under the PRISMA guidelines.

\section{Results and Discussions}

GSC is typically identified after 2 years from the surgical intervention which implied partial resection of the stomach due to a malignant tumor. Cancer development in the gastric stump after benign surgery has an increased latency of up to 20 years as reported by Lagergen et al. and Lee et al. [6] [7]. Such a substantial difference in time between benign and malignant up to 18 years is due to the fact that precancerous microscopic lesions which have led to the development of the primary tumor are already present in the neoplastic stomach such as intestinal metaplasia or atrophic gastritis [8]. These cellular abnormalities develop at distance from the tumor and usually are not included in the resected specimen [8]. Also, the follow-up protocol is more rigorous after malignant lesions than benign lesions and allows early identification [8]. Some studies advocate that the immunologic response which develops from the surgical stress or associated with infection may maintain viable residual tumoral cells, which results in neoplasia recurrence [9] [10].

After the development of anti-secretory molecules for gastro-duodenal ulcers, the number of gastrectomies for benign pathologies has markedly diminished, but the incidence of GCS did not decrease, as a result of the long 
period of carcinogenesis of up to 20 years [11][12].

Regarding the etiology of GSC, important anatomical and physiological modifications occur after partial gastrectomies, the mucosa is exposed for a prolonged interval of time to biliary reflux. The increase of the gastric $\mathrm{pH}$, especially at the anastomotic site is considered a crucial factor for chronic gastric inflammation which in time leads to metaplasia and eventually cancer [13].

Nowadays, the sedentary lifestyle and unhealthy eating-habits had produced an increased incidence of obesity to the point that is has become a global health problem, with an incidence reaching even $30 \%$ in western countries. This has led to a significant rise in bariatric procedures which frequently imply the partial resection of the stomach. In 2019, Tornese et al. conducted a systematic literature review and identified 17 cases of GSC after gastric bypass with Roux-en-Y [14].

The study published by Ohashi et al. revealed that in 50 patients with GSC after partial gastrectomies due to benign and malignant lesions, only 3 cases were located at the level of the gastric suture, 43 cases at distance from the suture sites and 4 cases in which the tumor involved the entire remaining stomach [15]. The same author mentions that the development of the neoplasia at the level of the suture site has a worse prognosis, but this theory is still a debatable subject since other authors assert the contrary [3] [16][17][18].

The main route of dissemination of GSC as for the primary gastric tumor is lymphatic. Surgical intervention varies accordingly to the type of gastrectomy previously performed and tumor histopathologic characteristics which frequently impose an extended lymphadenectomy. One must take into account that the postoperative anatomical variations lead to a change of the normal lymphatic drainage pattern which is mostly directed to the ganglia of the splenic artery and hilum [19][20][21]. The article published by Iguchi et al. demonstrated that after Billroth II gastrojejunostomy during index surgery, lymph node metastases can be found as far as the jejunal mesentery. Therefore, the author mentions that jejunal mesentery lymphadenectomy for GSC is mandatory [22]. This recommendation is open to debate due to the important morbidity it implies. J. Lu et al. published an article in 2014 which reported that the most important factors of prognosis for GSC independent of the surgery performed are the TNM stage and the $\mathrm{N}$ group stage [23][24].

The retrospective study conducted by Takahashi et al. published in 2019, which included 122 patients from a period of 40 years showed that postoperative morbidity may reach $18.9 \%$. Anastomosis fistula was the most frequent encountered complication [25]. An incriminating factor may have been the neoplastic status of patients which frequently leads to increased consumptions syndromes and denutrition. Preoperative evaluation and proper nutrition status are mandatory for these high-risk patients.

Advances in postoperative follow-up programs have led to an increased rate of diagnosis in early stages of GSC. This, in turn, has to lead to an increased rate of respectability of these tumors which has increased the 5-year overall survival rate. An important role for this rise is attributed to the progress of chemotherapy and especially to immunotherapy [26]. Thus, in 2000 Thorbadan et al. noted a 5-year survival rate between 3 and 10\%, whereas in 2019 the 5-year overall survival rate reported by Leo et al. was $53.1 \%$ and St.-Louis et al. of 58\% [16] [27].

\section{Conclusion}

More studies are required to better understand the mechanism of development and the evolution of GSC. The published articles were conducted on a small number of patients; thus, the information is not yet enough to elaborate clear guidelines for the treatment of GSC. With these aspects in mind, an increase in the 5-year overall survival rate has been observed, which has been attributed to improved follow-up after index surgery, enhanced imagistics and diagnosis at an early stage. For the future, we need to observe closely the impact that bariatric surgery has on the incidence of gastric cancer as the world population continues the increasing trend of obesity. 


\section{Conflicts of interests}

The authors declare no conflicts of interest.

\section{Authors Contributions}

Conception and design: Iulian Slavu, Adrian Tulin, Lucian Alecu, Braga Vlad, Bogdan Socea Provision of study material or patients: Adrian Tulin, Lucian Alecu, Mihaila Daniela

Collection and assembly of data: Iulian Slavu, Adrian Tulin, Lucian Alecu, Slavu Iulian

Data analysis and interpretation: Iulian Slavu, Alecu Lucian, Braga Vlad

Manuscript writing: Iulian Slavu, Cornelia Nitipir, Braga Vlad, Bogdan Socea

Final approval of manuscript: Lucian Alecu, Cornelia Nitipir, Adrian Tulin

\section{References}

[1] Balfour D C 1922 FACTORS INFLUENCING THE LIFE EXPECTANCY OF PATIENTS OPERATED ON FOR GASTRIC ULCER Ann. Surg.76 405-8

[2] Li F, Zhang R, Liang H, Zhao J, Liu H, Quan J, Wang X and Xue Q 2013 A Retrospective Clinicopathologic Study of Remnant Gastric Cancer After Distal Gastrectomy Am. J. Clin. Oncol.36 2449

[3] Irino T, Hiki N, Ohashi M, Nunobe S, Tokunaga M, Sano T and Yamaguchi T 2016 Characteristics of gastric stump cancer: A single hospital retrospective analysis of 262 patients Surg. (United States) 159 1539-47

[4] Nienhüser H, Blank S, Sisic L, Kunzmann R, Heger U, Ott K, Büchler M W, Schmidt T and Ulrich A 2017 [Gastric stump carcinoma: frequency, treatment, complications and prognosis]. Chirurg. 88 317-27

[5] Diogo Filho A, Botelho L F, Nishiyama A, Zumpano L E, Monte R C, Rosa S C and ROSA S C 2016 GASTRIC STUMP CANCER AFTER GASTRECTOMY BY GASTRODUODENAL PEPTIC ULCER. Arq. Bras. Cir. Dig.29 65

[6] Lagergren J, Lindam A and Mason R M 2012 Gastric stump cancer after distal gastrectomy for benign gastric ulcer in a population-based study Int. J. Cancer131 1048-52

[7] Lee S B, Kim J H, Kim D H, Jeon T Y, Kim D H, Kim G H and Park D Y 2010 Clinicopathological characteristics and prognosis of remnant gastric cancer. J. Gastric Cancer10 219-25
[8] Ohira M, Toyokawa T, Sakurai K, Kubo N, Tanaka H, Muguruma K, Yashiro M, Onoda N and Hirakawa K 2016 Current status in remnant gastric cancer after distal gastrectomy World J. Gastroenterol.22 2424

[9] Maehara Y, Tsujitani S, Saeki H, Oki E, Yoshinaga K, Emi Y, Morita M, Kohnoe S, Kakeji Y, Yano T and Baba H 2012 Biological mechanism and clinical effect of protein-bound polysaccharide $\mathrm{K}$ (KRESTIN (®)): review of development and future perspectives. Surg. Today42 8-28

[10] Hayashi T, Yoshikawa T, Aoyama T, Hasegawa S, Yamada T, Tsuchida K, Fujikawa H, Sato T, Ogata T, Cho H, Oshima T, Rino Y and Masuda M 2015 Impact of infectious complications on gastric cancer recurrence Gastric Cancer18 368-74

[11] Behrman S W 2005 Management of Complicated Peptic Ulcer Disease Arch. Surg.140 201

[12] Komatsu S, Ichikawa D, Okamoto K, Ikoma D, Tsujiura M, Nishimura Y, Murayama Y, Shiozaki A, Ikoma H, Kuriu Y, Nakanishi M, Fujiwara H, Ochiai T, Kokuba Y and Otsuji E 2012 Progression of remnant gastric cancer is associated with duration of follow-up following distal gastrectomy. World $\mathrm{J}$. Gastroenterol.18 2832-6

[13] Păduraru D N, Nica A, Ion D, Handaric M and Andronic O 2016 Considerations on risk factors correlated to the occurrence of gastric stump cancer. J. Med. Life9 130-6

[14] Tornese S, Aiolfi A, Bonitta G, Rausa E, Guerrazzi G, Bruni P G, Micheletto G and Bona D 2019 Remnant Gastric Cancer After Roux-en-Y Gastric Bypass: Narrative Review of the Literature Obes. Surg. 1-5

[15] Ohashi M, Morita S, Fukagawa T, Kushima R and Katai H 2015 Surgical treatment of non-early gastric remnant carcinoma developing after distal gastrectomy for gastric cancer J. Surg. Oncol.111 $208-12$

[16] Thorban S, Böttcher K, Etter M, Roder J D, Busch R and Siewert J R 2000 Prognostic factors in gastric stump carcinoma. Ann. Surg.231 188-94

[17] Chen L, Wei S, Ye Z, Wang Y, Zheng Q, Zhuo C, Xiao J and Zeng Y 2017 [Study on the clinicopathologic characteristics and prognostic difference of gastric stump cancer between nonanastomotic site and anastomotic site]. Zhonghua Wei Chang Wai Ke Za Zhi20 67-72

[18] Cheng K-S, Tang H-L, Chou J-W, Yu C-J, Tsou S-S and Chou F-T 2011 The clinical features and prognosis of gastric remnant carcinoma after treatment. ISRN Gastroenterol.

[19] Nakagawa M, Choi Y Y, An J Y, Hong J H, Kim J W, Kim H-I, Cheong J-H, Hyung W J, Choi S H and Noh S H 2016 Staging for Remnant Gastric Cancer: 
The Metastatic Lymph Node Ratio vs. the UICC 7th Edition System Ann. Surg. Oncol.23 4322-31

[20] Aoyagi K, Kouhuji K, Miyagi M, Imaizumi T, Kizaki J and Shirouzu K 2010 Prognosis of metastatic splenic hilum lymph node in patients with gastric cancer after total gastrectomy and splenectomy. World J. Hepatol.2 81-6

[21] Honda S, Bando E, Makuuchi R, Tokunaga • Masanori, Tanizawa Y, Kawamura • Taiichi, Sugiura

T, Kinugasa Y, Katsuhiko Uesaka • and Terashima • Masanori Effects of initial disease status on lymph flow following gastrectomy in cases of carcinoma in the remnant stomach

[22] Iguchi K, Kunisaki C, Sato S, Tanaka Y, Miyamoto H, Kosaka T, Akiyama H, Endo I, Rino Y and Masuda M 2018 Evaluation of Optimal Lymph Node Dissection in Remnant Gastric Cancer Based on Initial Distal Gastrectomy Anticancer Res.38 167783

[23] Lu J, Huang C, Zheng C, Li P, Xie J, Wang J, Lin J, Chen Q, Cao L and Lin M 2014 Prognostic Value of Tumor Size in Patients with Remnant Gastric Cancer: Is the Seventh UICC Stage Sufficient for Predicting Prognosis? ed Q-Y Wei PLoS One9 e115776

[24] Kameda C, Kawabata R, Koga C, Matsumura T, Murakami M, Noura S, Shimizu J and Hasegawa J 2019 Clinicopathological Features of Remnant Gastric Cancer (RGC): Detection of RGC after Five Years of Follow-Up Was Associated with a Poor Prognosis Am. Surg.85 384-9

[25] Takahashi M, Takeuchi H, Tsuwano S, Nakamura R, Takahashi T, Wada N, Kawakubo H, Saikawa Y and Kitagawa Y 2016 Surgical Resection of Remnant Gastric Cancer Following Distal Gastrectomy: A Retrospective Clinicopathological Study Ann. Surg. Oncol.23 511-21

[26] Takeno S, Hashimoto T, Maki K, Shibata R, Shiwaku H, Yamana I, Yamashita R and Yamashita Y 2014 Gastric cancer arising from the remnant stomach after distal gastrectomy: a review. World J. Gastroenterol.20 13734-40

[27] St-Louis E, Gowing S D, Mossallanejad P, Leimanis M L, Mueller C and Ferri L E 2018 Outcomes after completion total gastrectomy for gastric remnant cancer: experience from a Canadian tertiary centre. Can. J. Surg.61 270-7 